\title{
PREPARAÇÃO DE $N$-ALQUIL DERIVADOS DO 1-BROMO-2,4-DINITROBENZENO E DO 1-CLORO-2- NITROBENZENO. UMA ALTERNATIVA ÀS AULAS PRÁTICAS DE SUBSTITUIÇÃO NUCLEOFÍLICA AROMÁTICA
}

\author{
Ayres Guimarães Dias*, Felipe Martins Alves Pereira e Renato de Oliveira Soares \\ Departamento de Química Orgânica, Instituto de Química, Universidade do Estado do Rio de Janeiro, R. São Francisco Xavier, \\ 524, Pavilhão Haroldo Lisboa, 20559-900 Rio de Janeiro - RJ, Brasil
}

Recebido em 20/6/07; aceito em 29/2/08; publicado na web em 1/10/08

\begin{abstract}
PREPARATION OF $N$-ALKYL DERIVATIVES OF 1-BROMO-2,4-DINITROBENZENE AND 1-CHLORO-2-NITROBENZENE. AN ALTERNATIVE TO NUCLEOPHILIC AROMATIC SUBSTITUTION PRACTICES. Preparation of the title compounds are described as an alternative nucleophilic aromatic substitution for practices in the graduate laboratory. The low toxicity and disponibility of the reagents make a suitable procedure approach to experiments regarding this aromatic reaction.
\end{abstract}

Keywords: aromatic nucleophilic substitution; nitrogenated nucleophiles; chemical education.

\section{INTRODUÇÃo}

O conteúdo programático das disciplinas práticas oferecidas pelos departamentos de química orgânica nos cursos de química, engenharia química e farmácia, a partir do terceiro período, é normalmente dedicado a experimentos ilustrativos incluindo a reatividade do anel aromático. ${ }^{1}$ A reação de substituição nucleofílica em composto aromáticos é normalmente exemplificada com haletos de arila contendo grupos fortemente elétron-atraentes e espécies nucleofílicas centradas em heteroátomos, como nitrogênio e oxigênio. $\mathrm{O}$ grupo nitro é o principal grupo substituinte empregado, devido a sua grande capacidade de estabilização do intermediário aniônico formado e sua facilidade de preparação. ${ }^{2}$ A reação tem amplo emprego analítico, sendo bastante utilizada na caracterização de aminoácidos terminais de proteínas e na quantificação de fármacos de estrutura peptídica, como o Lisinopril. ${ }^{3,4}$ Recentemente a substituição nucleofílica aromática foi utilizada na elucidação do papel catalítico dos aminoácidos presentes nos sítios ativos da Glutationa S transferase humana, hGMTS1-1, pela análise por difratometria de raio $\mathrm{X}$ do complexo enzima-substrato formado. ${ }^{5}$ O 1-flúor-2,4-dinitrobenzeno, DNFB, reagente de Sanger, é o halodinitrobenzeno de escolha na derivatização de materiais de origem biológica devido a sua maior reatividade, porém o alto custo do reagente limita seu emprego (Esquema 1). ${ }^{3,4}$ Todas as utilizações descritas estão baseadas na reação dos heteroátomos presentes nas cadeias laterais dos aminoácidos de proteínas e dos fármacos, através da reação de substituição nucleofílica do átomo de halogênio (Esquema 1). A fácil observação espectrofotométrica na região UV-VIS do espectro eletromagnético oriunda da alta absortividade molar deste fragmento molecular e o baixo custo dos halo-2,4-dinitrobenzenos, brometo e cloreto, torna esta estratégia uma alternativa analítica eficaz para químicos e bioquímicos.

Para exemplificar as reações envolvendo o mecanismo de substituição nucleofílica aromática nos cursos de graduação é utilizado o deslocamento pela hidrazina do 1-bromo-2,4-dinitrobenzeno ( 80\% de rendimento), normalmente preparado pelo próprio aluno usando a seqüência halogenação-nitração. ${ }^{6}$

Apesar de possuir a nucleofilicidade adequada ao processo, a alta toxicidade da hidrazina mono-hidratada e suas soluções tornam

*e-mail: ayres@uerj.br

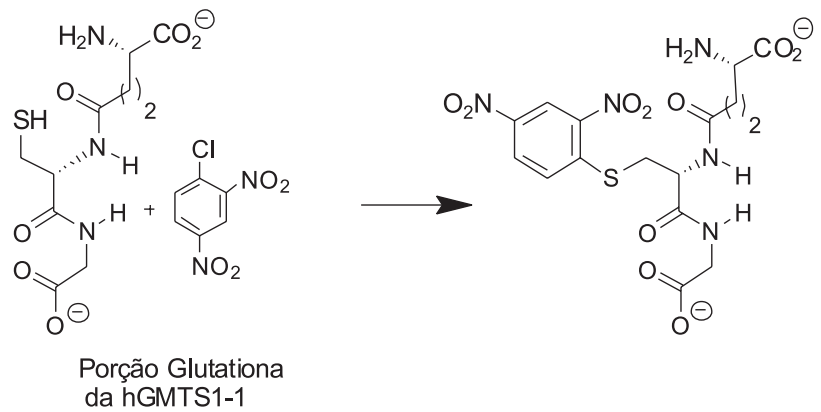

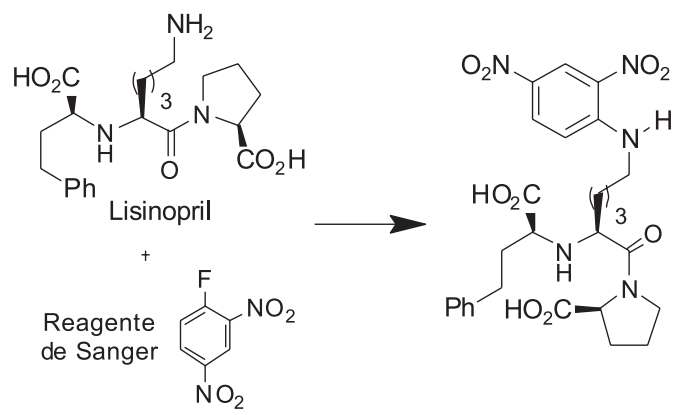

Esquema 1

a manipulação uma operação inadequada aos alunos dos primeiros semestres do ciclo básico. ${ }^{7}$ Recentemente, o produto teve sua venda interrompida durante alguns meses devido a dificuldades de importação. O emprego de soluções mais diluídas de hidrazina, ainda disponíveis no mercado ( 50\%), não foi capaz de promover a reação com o 1-bromo-2,4-dinitrobenzeno e as práticas e monografias envolvendo o uso desse reagente foram suspensas.

Das opções descritas na literatura para este tipo de reação, poucas são capazes de satisfazer as necessidades inerentes a uma aula, como o tempo e forma de purificação. ${ }^{6} \mathrm{Na}$ busca de experimentos de baixo custo compatíveis com as condições existentes em um laboratório de graduação no país, optamos pela substituição da hidrazina por três espécies nucleofílicas cuja disponibilidade comercial e toxicidade não inviabilizassem seu uso. A morfolina, o imidazol e a etanolamina foram eleitos por preencherem todos os requisitos desejados e estarem disponíveis no mercado nacional a baixo custo. ${ }^{7} \mathrm{O}$ produto de substi- 
tuição com a etanolamina, $(\mathbf{3 c})$, está estruturalmente relacionado com o corante semi-permanente $\mathrm{HC}$ amarelo 2, 2-(2-nitrofenilamino)etanol, (5), empregado em preparações cosméticas capilares. ${ }^{8}$ A inclusão de $\mathbf{( 5 )}$ neste trabalho visou promover a comparação da reatividade em mecanismos de substituição nucleofílica aromática em anéis menos ativados, como o o-cloronitrobenzeno, (4) e contextualizar a importância industrial desta reação (Esquema 2). ${ }^{8}$

\section{RESULTADOS E DISCUSSÃO}

O 1-bromo-2,4-dinitrobenzeno foi deixado sob condições adaptadas dos experimentos de Rossi e Buján para o respectivo cloro derivado. As reações mostraram-se extremamente reprodutíveis, de fácil execução em variações de escala de 1 a $10 \mathrm{~g}$, por agitação da mistura reacional por cerca de $1 \mathrm{~h}$ em DMF., ${ }^{9}, 10$ Um ligeiro aquecimento foi necessário para o deslocamento com imidazol (Esquema 2). Os produtos brutos de isolamento, após secagem, foram caracterizados por infravermelho (IV), espectrometria de massas (EM) e ressonância magnética nuclear (RMN). A cromatografia de camada fina pode ser empregada no acompanhamento da reação e, em conjunto com o ponto de fusão, serve como uma eficiente ferramenta de caracterização em laboratórios que não possuam equipamentos mais sofisticados.

Morfolina e imidazol mostraram-se capazes de substituir a hidrazina como espécie nucleofílica, em reações com o 1-bromo-2,4dinitrobenzeno. Os experimentos mostraram-se pouco influenciados por alterações de escala e de operador, tendo os produtos sido obtidos de forma adequada a uma aula prática de graduação.

A etanolamina apresentou reatividade similar aos outros compostos aminados quando submetida às condições de Rossi e Buján, porém mostrou-se de difícil isolamento devido a sua solubilidade parcial em água. O emprego da metodologia de Jatzkewitz solucionou o problema com (3c) sendo obtido em bons rendimentos após $46 \mathrm{~h}$ de reação. ${ }^{11} \mathrm{O}$ corante HC Amarelo 2, (5), pode ser isolado após $4 \mathrm{~h}$ de aquecimento (80-90 ${ }^{\circ} \mathrm{C}$ ), com catálise de $\mathrm{CuCl}_{2}$, como descrito por McManus, somente em escalas próximas à relatada pelo autor. ${ }^{12} \mathrm{~A}$ diferença no tempo e temperatura de reação é um importante elemento comparativo sobre a reatividade dos anéis aromáticos mono e dissubstituídos e deve

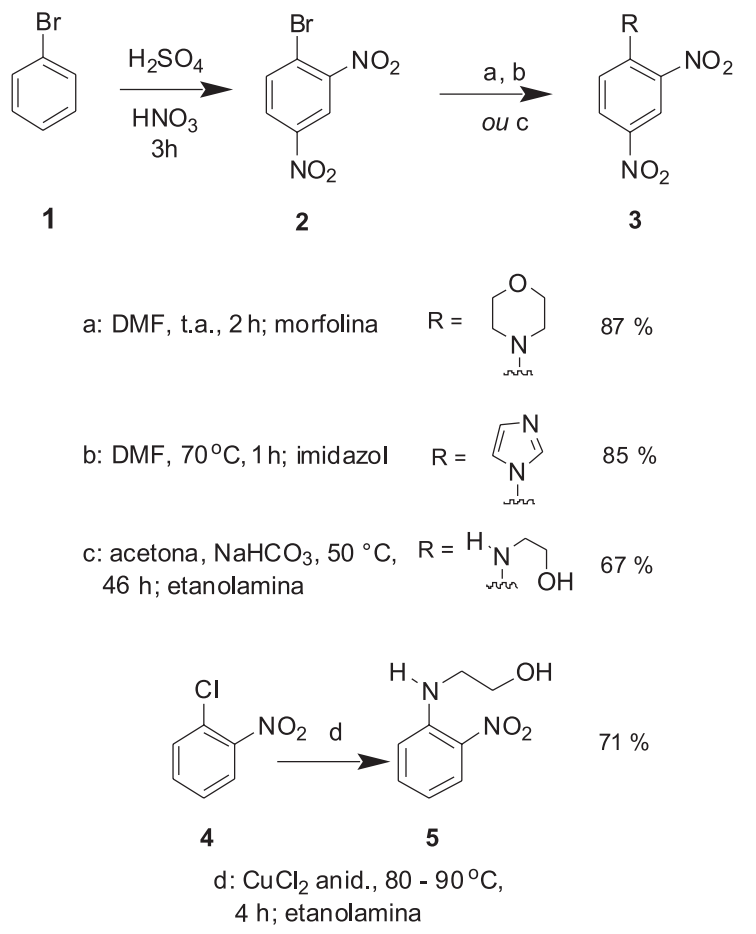

Esquema 2 ser explorado pelo professor como tema motivador.

A discussão sobre as características das espécies nucleofílicas empregadas e a introdução de compostos heterocíclicos nas práticas das disciplinas básicas do curso de química orgânica experimental aqui propostas podem também contribuir para uma contextualização desta importante família de compostos orgânicos e servir como uma alternativa às questões didáticas envolvendo o ensino de química experimental. ${ }^{13-17}$

\section{PARTE EXPERIMENTAL}

Os pontos de fusão foram determinados em aparelho Buchi 530 e devidamente corrigidos. Os espectros na região do infravermelho, (IV), foram registrados em pastilhas de $\mathrm{KBr}\left(v, \mathrm{~cm}^{-1}\right)$, em um espectrômetro Nicolet - Magna FT-IR 760, na região de 4000 a $600 \mathrm{~cm}^{-1}$. Os espectros de ressonância magnética nuclear de hidrogênio e carbono, (RMN ${ }^{1} \mathrm{H} \mathrm{e}{ }^{13} \mathrm{C}$ ), foram obtidos em espectrômetro Varian Gemini $200(200 \mathrm{MHz})$. Os deslocamentos químicos estão apresentados em partes por milhão $(\delta)$, relativos ao tetrametilsilano (TMS, $\delta=0,0$ ); como solvente utilizou-se $\mathrm{CDCl}_{3}$ e DMSO-d $\mathrm{d}_{6}$. Os espectros de massas, (EM), foram obtidos em espectrômetro HP CG-EM 5973, operando a $70 \mathrm{eV}$. O sulfato de cobre anidro foi obtido pelo aquecimento do $\mathrm{CuSO}_{4} \cdot 6 \mathrm{H}_{2} \mathrm{O}$, a $110{ }^{\circ} \mathrm{C}$, por $6 \mathrm{~h}^{18}$

\section{Procedimentos sintéticos}

$N$-(2,4-dinitrofenil)-morfolina (3a)

Em um balão de $25 \mathrm{~mL}$, envolvido em papel alumínio, contendo 1,3 g (5,3 mmol) de 1-bromo-2,4-dinitrobenzeno, dissolvido em $10 \mathrm{~mL}$ de DMF, foi adicionado 1,0 mL (11,5 mmol) de morfolina. A solução foi agitada à temperatura ambiente por $2 \mathrm{~h}$ e vertida sobre água fria. $\mathrm{O}$ precipitado foi filtrado a vácuo e seco em dessecador, dando origem a 1,2 g (87\% rendimento) de um sólido amarelo, de ponto de fusão $118,0-119,0^{\circ} \mathrm{C}$ (p.f. lit. ${ }^{10,11}=117,0-118,0^{\circ} \mathrm{C}$ ), $\mathrm{R}_{\mathrm{f}}=0,66$, usando uma mistura acetato de etila/hexano como eluente e iodo como revelador. ${ }^{19,20}$ IV $\left(\mathrm{cm}^{-1}\right) v_{\max }: 3116,3091,2987,2919,2864,1605,1531,1501,1322$. RMN- ${ }^{1} \mathrm{H}\left(\mathrm{CDCl}_{3}, 200 \mathrm{MHz}\right) \delta: 3,31(\mathrm{t}, 4 \mathrm{H}, J=4,7 \mathrm{~Hz}), 3,88$ (t, 4H, $J=4,7 \mathrm{~Hz}), 7,16(\mathrm{~d}, 1 \mathrm{H}, J=9,3 \mathrm{~Hz}), 8,22(\mathrm{dd}, 1 \mathrm{H}, J=2,8 \mathrm{~Hz} J=9,3$ $\mathrm{Hz}), 8,66(\mathrm{~d}, 1 \mathrm{H}, J=2,8 \mathrm{~Hz}) . \mathrm{RMN}-{ }^{13} \mathrm{C}\left(\mathrm{CDCl}_{3}, 50 \mathrm{MHz}\right), \delta: 50,58(2$ CH2), 65,84 (2 CH2), 118,98 (CH), 123,30 (CH), 128,08 (CH), 137,91 (Cquat), 138,33 (Cquat), 149,05 (Cquat).

\section{1-(2,4-dinitrofenil)-imidazol (3b)}

Em um balão de $50 \mathrm{~mL}$, acoplado a um condensador de refluxo, envolvido em papel de alumínio e contendo 4,9 g (20,0 mmol) de 1-bromo-2,4-dinitrobenzeno dissolvidos em 20,0 mL de DMF foram adicionados 2,7 g (39,7 mmol) de imidazol. A solução foi aquecida a $70{ }^{\circ} \mathrm{C}$ por $1 \mathrm{~h}$ e vertida sobre água fria. $\mathrm{O}$ precipitado foi filtrado a vácuo e seco em dessecador, dando origem a $1,98 \mathrm{~g}$ ( $85 \%$ rendimento) de um sólido amarelo alaranjado, de ponto de fusão $144,0^{\circ} \mathrm{C}$, (p.f. lit. $.^{10,11}=144,0$ $\left.{ }^{\circ} \mathrm{C}\right), \mathrm{R}_{\mathrm{f}}=0,33$, usando uma mistura acetato de etila/hexano como eluente e iodo como revelador. ${ }^{19,20} \mathrm{IV}\left(\mathrm{cm}^{-1}\right) \mathrm{v}_{\max }: 3122 ; 3033 ; 3010 ; 1606,1547$, $1530,1513,1350,1337 . \mathrm{RMN}^{-} \mathrm{H}\left(200 \mathrm{MHz}, \mathrm{CDCl}_{3}, \mathrm{TMS}\right) \delta: 7,17$ (s, 1H), 7,53 (t, 1H, J=1,2 Hz), 8,03 (d, 1H, $J=8,8 \mathrm{~Hz}), 8,05$ (s, 1H), $8,63(\mathrm{dd}, 1 \mathrm{H}, J=2,6 \mathrm{~Hz} J=8,8 \mathrm{~Hz}) ; 8,95(\mathrm{~d}, 1 \mathrm{H}, J=2,6 \mathrm{~Hz}) . \mathrm{RMN}-{ }^{13} \mathrm{C}$ (DMSO, $50 \mathrm{MHz}), \delta$ : 120,33 (CH), 121,23 (CH),128,67 (CH), 129,84 (CH), 130,16(CH), 134,86 (Cquat), 137,45 (CH), 143,76 (Cquat), 146,42 (Cquat). EM m/z (\%): 234 (13), 179(80), 103(100).

\section{2-(2,4-dinitrofenilamino)-etanol (3c)}

Em um balão de $25 \mathrm{~mL}$, acoplado a um condensador de refluxo envolvido em papel alumínio contendo 1,0 g (4,1 mmol) de 1-bromo-2,4 dinitrobenzeno e $1,5 \mathrm{~mL}$ de etanolamina $(24,6 \mathrm{mmol})$ foram adicionados $10,0 \mathrm{~mL}$ de uma solução $8,5 \%$ de $\mathrm{NaHCO}_{3}(10,1 \mathrm{mmol})$ 
e $20,0 \mathrm{~mL}$ de acetona. A solução foi agitada a $50{ }^{\circ} \mathrm{C}$ por $46 \mathrm{~h}$. Após separação da fase orgânica, a mistura reacional foi extraída 3 vezes com $15 \mathrm{~mL}$ de éter etílico e as frações orgânicas foram reunidas e concentradas em rotaevaporador. $\mathrm{O}$ produto foi submetido à cromatografia em coluna cromatográfica de gel de sílica com éter etílico como eluente $(100 \mathrm{~mL})$ e após evaporação deu origem a $0,6 \mathrm{~g}$ de um sólido castanho avermelhado de ponto de fusão $89-90{ }^{\circ} \mathrm{C}(67 \%$ de rendimento). Após recristalização em uma mistura etanol-água o ponto de fusão alterou-se para $89,5-90,5^{\circ} \mathrm{C}$; (p.f. lit. ${ }^{11,21}=89,0-90,0$ $\left.{ }^{\circ} \mathrm{C}\right), \mathrm{R}_{\mathrm{f}}=0,33$, usando uma mistura acetato de etila/hexano $1: 1$ como eluente e iodo como revelador. ${ }^{11,19,21} \mathrm{IV}\left(\mathrm{cm}^{-1}\right) v_{\max }: 3334,3105,2952$, $1620,1583,1520,1500,1338,1307 . \mathrm{RMN}^{-}{ }^{\mathrm{H}} \mathrm{H}\left(200 \mathrm{MHz}, \mathrm{CDCl}_{3}\right.$, TMS) $\delta: 9,15(\mathrm{~d}, 1 \mathrm{H}, J=2,6 \mathrm{~Hz}), 8,92-8,82(\mathrm{sl}, 1 \mathrm{H}), 8,24(\mathrm{dd}, 1 \mathrm{H}$, $J=2,6 \mathrm{~Hz} J=9,6 \mathrm{~Hz}$ ), 7,00 (d, 1H, $J=9,6 \mathrm{~Hz}$ ), 4,03 (t, 2H, $J=5,4$ $\mathrm{Hz}) ; 3,62$ (q, 2H, $J=5,4 \mathrm{~Hz}), 1,6-2,1(\mathrm{sl}, 1 \mathrm{H}) . \mathrm{RMN}-{ }^{13} \mathrm{C}\left(\mathrm{CDCl}_{3}\right.$, $50 \mathrm{MHz}$ ), $\delta: 148,44$ (Cquat), 136,02 (Cquat),130,47 (Cquat), 130,23 $(\mathrm{CH}), 124,22(\mathrm{CH}), 113,95(\mathrm{CH}), 60,34(\mathrm{CH} 2), 45,08(\mathrm{CH} 2) . \mathrm{EM}$ $\mathrm{m} / \mathrm{z}$ (\%): 228 (2); 227 (14), 196(100), 77(21)

\section{2-(2-nitrofenilamino)-etanol (5)}

A uma solução de o-cloronitrobenzeno $(27,5 \mathrm{~g} ; 0,18 \mathrm{~mol}) \mathrm{em}$ $190,0 \mathrm{~mL}$ de etanolamina $(3,14 \mathrm{~mol})$ foram adicionados $4,5 \mathrm{~g}$ de $\mathrm{CuCl}_{2}$ anidro $(0,03 \mathrm{~mol})$ - obtido após secagem do cloreto hidratado comercial em estufa a $130{ }^{\circ} \mathrm{C}$ por $8 \mathrm{~h}$. A mistura reacional foi aquecida em banho de óleo por $4 \mathrm{~h}$, com o cuidado de não deixar que a temperatura ultrapassasse $90^{\circ} \mathrm{C}$. A mistura racional foi então vertida em $400 \mathrm{~mL}$ de água e mantida sobre refrigeração em geladeira ou banho de gelo até precipitação. Após a filtração e secagem foi obtido um sólido laranja-avermelhado, $31,0 \mathrm{~g}$ (71\% de rendimento), de ponto de fusão $71,5-73,0^{\circ} \mathrm{C}$. A recristalização em uma mistura etanol-água deu origem a um sólido de ponto de fusão $72,5-73,5^{\circ} \mathrm{C}$; (p.f. lit. ${ }^{12}=$ $\left.72,5-73,5^{\circ} \mathrm{C}\right), \mathrm{R}_{\mathrm{f}}=0,42$ usando uma mistura acetato de etila/hexano $1: 1$ como eluente e iodo como revelador. ${ }^{12}$

IV $\left(\mathrm{cm}^{-1}\right) v_{\max }: 3339,3020,2960,1626,1569,1511,1309 . \mathrm{RMN}-$ ${ }^{1} \mathrm{H}\left(200 \mathrm{MHz}, \mathrm{CDCl}_{3}\right.$, TMS) $\delta: 8,16-8,28(\mathrm{sl}, 1 \mathrm{H}), 8,08(\mathrm{dd}, 1 \mathrm{H}$, $J=1,4 \mathrm{~Hz} J=8,3 \mathrm{~Hz}), 7,35-7,48(\mathrm{~m}, 1 \mathrm{H}), 6,85(\mathrm{~d}, 1 \mathrm{H}, J=8,6 \mathrm{~Hz})$, 6,55-6,68 (m, 1H); 3,92 (t, 2H, $J=5,2 \mathrm{~Hz}) .3,47$ (q, 2H, $J=5,2 \mathrm{~Hz}$ ), $2,80$ - 3,14 (sl, $1 \mathrm{H}) . \mathrm{RMN}^{13} \mathrm{C}\left(\mathrm{CDCl}_{3}, 50 \mathrm{MHz}\right), \delta: 145,32$ (Cquat), 136,08 (CH), 131,68 (Cquat), 126,59 (CH), 115,27 (CH), 113,57 (CH), 60,44 (CH2), 44,70 (CH2). EM m/z (\%): 183 (3), 182 (26); 151(100), 93(35)

\section{AGRADECIMENTOS}

À Central Analítica do NPPN-UFRJ e à Dra. C. Resende do IQUFRJ pelos espectros dos produtos obtidos.

\section{REFERÊNCIAS E NOTAS}

1. Zucco, C.; Pessine, F. B. T.; de Andrade, J. B.; Quim. Nova 1999, 22, 454.

2. Ono, N.; The Nitro Group in Organic Synthesis, John Wiley \& Sons: New York, 2001, cap. 9.
3. Sanger, F.; Biochem. J. 1945, 39, 507; De Bruin, S. H.; Bucci, E.; J. Biol. Chem. 1971, 246, 5228 .

4. Paraskevas, G.; Atta-Politou, J.; Koupparis, M.; J. Pharm. Biomed. Anal. 2002, 29, 865; Para uma discussão detalhada sobre a reatividade dos diferentes tipos de halogênios ver Simth, M. B. ; March, J.; Advanced Organic Chemistry, $5^{\text {th }}$ ed., John Wiley \& Sons: New York, 2000, cap. 13.

5. Patskovsky, Y.; Patskovska, L.; Almo, S. C.; Litstowsky, I.; Biochemistry 2006, 45, 3852 .

6. A seqüência de bromação-dinitração é de execução menos trabalhosa devido ao maior ponto de fusão do intermediário bromado, ainda que a reatividade na reação de deslocamento seja ligeiramente superior com clorodinitrobenzeno. Vogel, A. I.; Vogel's Textbook on Experimental Organic Chemistry, Longmann: Essex, 1989, cap. 6; Graham, K. J.; Schaller, C. P.; Johnson, B. J.; Klassen, J. B.; Chemical Educator 2002, 7, 376 .

7. Hidrazina $\mathrm{LD}_{50}=25 \mathrm{mg} / \mathrm{kg}$. Ação extremamente cáustica sobre a pele, mucosa ou membrana. Pode causar injúrias ao fígado e ao sangue. Carcinogênico em camundongos e ratos. Imidazol, $\mathrm{LD}_{50}=1880 \mathrm{mg} / \mathrm{kg}$; Morfolina $\mathrm{LD}_{50}=1050 \mathrm{mg} / \mathrm{kg}$; Etanolamina $\mathrm{LD}_{50}=1720 \mathrm{mg} / \mathrm{kg}$; The Merck Index; Windholz, M., ed.; $10^{\text {th }}$ ed., Merck \& Co. Inc.: New Jersey, 1983.

8. Anderson, J. A.; J. Soc. Dyers Colour 2000, 116, 193; Robbins, C. R.; Chemical and Physical Behavior of Human Hair, Springer-Verlag: New York, 2002, cap. 6; Navarro, M.; Sena, V. L. M.; Srivastava, R. M.; Navarro, D. M. A. F.; Quim. Nova 2005, 28, 1111.

9. de Vargas, E. B.; de Rossi, R. H.; J. Org. Chem. 1984, 49, 3978.

10. Remedi, M. V.; Buján, E. I.; Baggio, R.; Garland, M. T.; J. Phys. Org. Chem. 1998, 11, 895 .

11. A reação envolvendo a substituição nucleofílica em anéis menos ativados exige tempos superiores e deve ser empregada em práticas com alunos do programa de síntese orgânica; Jatzkewitz, H.; Tam, N. D. Z.; Physiol. Chem. 1954, 296, 188.

12. McManus, J. M.; Herbst, R. M.; J. Org. Chem. 1959, 24, 1042.

13. Bieber, L. W.; Quim. Nova 1999, 22, 605.

14. Srivastava, M. R.; Quim. Nova 1995, 18, 303.

15. Ferreira, V. F.; Silva, F. C.; Perrone, C. C.; Quim. Nova 2001, 24, 905.

16. Lagowski, J. M.; Katritzky, A. R Em Comprehensive Heterocyclic Chemistry; Katritzky, A. R., ed.; Pergamon Press: New York, 1984, vol. 5, cap. 4.01 .

17. Hull, L. A.; J. Chem. Educ. 2001, 78, 226; Horowitz, G.; J. Chem. Educ. 2007, 84, 346.

18. Perrin, D. D.; Armarego, W. L. F.; Purification of Laboratory Chemicals, Elsevier Science \& Technology Books: New York, 1988.

19. Os rendimentos descritos foram obtidos após três experimentos para cada tipo de produto sem a necessidade de qualquer outro tipo de purificação. A diminuição no tempo de reação levou à mistura de produtos de difícil isolamento. Na parte experimental foram descritas as médias dos rendimentos alcançados.

20. Não foram observadas mudanças nos valores de ponto de fusão após recristalizações em uma mistura etanol-água para (3a) e (3b).

21. Forlani, L.; Boga, C.; Mazza, M.; Cavrini, V.; Andrisano, V.; Tetrahedron 1998, 54, 4647 